\title{
ON THE ALGEBRAIC STRUCTURES OF GRADED LIE ALGEBRAS OF SECOND ORDER
}

\author{
By Kenji Atsuyama
}

\section{$\S 0$. Introduction.}

In 1973, by using of generalized Jordan triple systems of second order (=Kantor systems), I. L. Kantor [4] has given the models of graded Lie algebras of second order with involutive automorphism $\tau$. In this note, we shall prove the converse, that is, if $\tau$ is an automorphism of a Lie triple system in a graded Lie algebra of second order such that $\tau^{2}=1$ (resp. -1 ), it characterizes the Kantor (resp. Freudenthal) system. We also give a simple connection between the two kinds of triple systems.

\section{$\S 1$. A characterization of Kantor and Freudenthal systems.}

We consider a graded Lie algebra of second order

$$
\begin{aligned}
& \mathbb{B}=\mathbb{G}_{-2} \oplus \mathbb{S}_{-1} \oplus \mathbb{S}_{0} \oplus \mathbb{S}_{1} \oplus \mathbb{S}_{2} \quad \text { (direct sum) } \\
& {\left[\mathbb{S}_{i}, \mathbb{S}_{j}\right] \subset \mathbb{G}_{i+\jmath}}
\end{aligned}
$$

over a field $k$ of characteristic zero. Then the vector space $\mathbb{S}_{-1} \oplus \mathbb{S}_{1}$ becomes a Lie triple system (L.t. s.) with a triple product $[[X, Y], Z]$ where $[$,$] is the$ Lie product of $\mathbb{S}$ and elements $X, Y, Z$ are in $\mathbb{S}_{-1} \oplus \mathbb{S}_{1}$ (cf. [7]). Let $\tau$ be an automorphism of the L.t.s. $\mathbb{S}_{-1} \oplus \mathbb{S}_{1}$ with respect to the triple product. Then $\tau$ is called an $\varepsilon$-structure on $\mathbb{S}_{-1} \oplus \mathbb{S}_{1}(\varepsilon= \pm 1)$ if $\tau^{2}=$ sid and $\tau\left(\mathbb{S}_{ \pm 1}\right)=\mathbb{G}_{\mp 1}$.

Let $V$ be a finite dimensional vector space over the field $k$. Then $V$ is called a Kantor (resp. Freudenthal) system (cf. [4], [2], [8]) if $V$ has a trilinear operation $\phi: V \times V \times V \rightarrow V$ such that

1) $[L(a, b), L(c, d)]=L(L(a, b) c, d)-\varepsilon L(c, L(b, a) d)$,

2) $K(K(a, b) c, d)=L(d, c) K(a, b)+\varepsilon K(a, b) L(c, d)$

for $a, b, c, d \in V$, where $L(a, b) c=\phi(a, b, c), K(a, b) c=L(a, c) b-L(b, c) a$ and $\varepsilon=1$ (resp. -1).

This research was partially supported by the Scientific Research Grant of the Ministry of Education, 1979-D-464031.

Received November 20, 1980 
Now let $\mathbb{S}$ be a graded Lie algebra of second order which form is of (1.1) and let $\tau$ be an $\varepsilon$-structure on the L.t.s. $\mathbb{S}_{-1} \oplus \mathbb{S}_{1}$. We denote by $\tau_{ \pm 1}$ the $\varepsilon$-structure $\tau$ restricted to $\mathbb{S}_{ \pm 1}$, but, for simplicity, we sometimes use the same notation $\tau$ instead of $\tau_{ \pm 1}$ unless the confusion does not occur. When we write an element $a+\tau(x)$ in $\mathbb{S}_{-1} \oplus \mathbb{S}_{1}$ as the column vector, the Lie triple product $[[a+\tau(x), b+\tau(y)], c+\tau(z)]$ in $\mathbb{S}_{-1} \oplus \mathbb{S}_{1}$ can be denoted by

$$
\left[\left(\begin{array}{c}
a \\
\tau(x)
\end{array}\right)\left(\begin{array}{c}
b \\
\tau(y)
\end{array}\right)\left(\begin{array}{c}
c \\
\tau(z)
\end{array}\right)\right]=\left(\begin{array}{l}
K(a, b) z+L(a, y) c-L(b, x) c \\
\varepsilon \tau(K(x, y) c+L(x, b) z-L(y, a) z)
\end{array}\right)
$$

for $a, b, c, x, y, z \in \mathbb{S}_{-1}$ where $L(a, b) c=[[a, \tau(b)], c]$ and $K(a, b) c=[[a, b], \tau(c)]$. Moreover, by using of $2 \times 2$ matrix forms and column vectors, the right side of (1.2) can be rewritten as the following form:

$$
\left(\begin{array}{cc}
L(a, y)-L(b, x) & K(a, b) \tau^{-1} \\
\varepsilon \tau K(x, y) & -\varepsilon \tau(L(y, a)-L(x, b)) \tau^{-1}
\end{array}\right)\left(\begin{array}{c}
c \\
\tau(z)
\end{array}\right)
$$

for $a, b, c, x, y, z \in \mathbb{S}_{-1}$.

If the Lie algebra $\mathbb{B}$ is semi-simple, it is isomorphic to the standard imbedding (Lie algebra) of the L.t.s. $\mathbb{S}_{-1} \oplus \mathbb{B}_{1}$ (see [6], [7]).

Now, let $V$ be any Kantor (resp. Freudenthal) system. Then the direct sum $\mathfrak{I}(V)=V \oplus V$ becomes a L.t. s. with an $\varepsilon$-structure $\tau$ by the trilinear multiplication of (1.2) where $\tau_{-1}=1$ and $\tau_{1}=\varepsilon$ with $\varepsilon=1$ (resp. -1 ). And, the standard imbedding (Lie algebra) $\mathfrak{L}(V)$ of the L.t.s. $\mathfrak{I}(V)$ has a structure of graded Lie algebra of second order (see [4], [8]). Then, we have the following.

THEOREM 1. Let (S) be a graded Lie algebra of second order which form is of (1.1). If the L.t.s. $\mathbb{S}_{-1} \oplus \mathbb{S}_{1}$ has a 1-structure (resp. -1-structure) $\tau$, $\mathbb{S}_{-1}$ is $a$ Kantor (resp. Freudenthal) system with respect to the trilinear operation $\phi(a, b, c)$ $=[[a, \tau(b)], c]$ for $a, b, c \in \mathbb{S}_{-1}$. Moreover, if $\mathbb{B S}$ is semi-simple, it is isomorphic to the standard imbedding (Lie algebra) $\mathfrak{L}\left(\mathbb{S}_{-1}\right)$ of the L.t. s. $\mathfrak{T}\left(\mathbb{S}_{-1}\right)$.

Proof. Assume that the L.t.s. $\mathbb{S}_{-1} \oplus \mathscr{G}_{1}$ in $\mathbb{B}$ has an $\varepsilon$-structure $\tau$. Then we show that $\mathbb{B}_{-1}$ is a Kantor or Freudenthal system with respect to the trilinear operation $\phi(a, b, c)(=L(a, b) c)=[[a, \tau(b)], c]$ corresponding to $\varepsilon=1$ or -1 respectively. The adjoint representation ad of the Lie algebra $\&$ is defined usually by $\operatorname{ad}(x) y=[x, y]$ for $x, y \in \mathbb{S}$.

First, for $a, b, c, d, e \in \mathbb{S}_{-1}$, we have

$$
\begin{aligned}
{[L(a, b), L(c, d)] } & =[\operatorname{ad}[a, \tau(b)], \operatorname{ad}[c, \tau(d)]] \\
& =\operatorname{ad}[[a, \tau(b)],[c, \tau(d)]] \\
& =\operatorname{ad}[[[a, \tau(b)], c], \tau(d)]+\operatorname{ad}[c,[[a, \tau(b)], \tau(d)]]
\end{aligned}
$$

by the Jacobi's identity. Since $[[a, \tau(b)], c]=L(a, b) c$ and $[[a, \tau(b)], \tau(d)]=$ 
$-\varepsilon \tau L(b, a) d$, the operation $\phi$ satisfies the axiom 1) of the triple system. Secondly, again by the Jacobi's identity, it holds that $[[a, b], \tau(c)]=L(a, c) b-L(b, c) a$, i. e., the definition of $K(a, b)$ in the Kantor or Freudenthal system coincides with the definition in the L.t.s. $\mathbb{S}_{-1} \oplus \mathbb{S}_{1}$ of (1.2). Then we can prove the axiom 2):

$$
\begin{aligned}
K(K(a, b) c, d) e & =[[[[a, b], \tau(c)], d], \tau(e)] \\
& =-[[[\tau(c), d],[a, b]], \tau(e)] \\
& =-\operatorname{ad}[[\tau(c), d],[a, b]] \tau(e) \\
& =-[\operatorname{ad}[\tau(c), d], \operatorname{ad}[a, b]] \tau(e) \\
& =-\operatorname{ad}[\tau(c), d] \operatorname{ad}[a, b] \tau(e)+\operatorname{ad}[a, b] \operatorname{ad}[\tau(c), d] \tau(e) \\
& =L(d, c) K(a, b) e+\varepsilon K(a, b) L(c, d) e
\end{aligned}
$$

where the second equality is proved by the Jacobi's identity and the relation $[[a, b], d] \in \mathbb{G}_{-3}=\{0\}$.

Now, let $\mathscr{S}_{-1}$ be the Kantor (resp. Freudenthal) system with the product $L(a, b) c$ which is obtained from $\mathbb{B}$ by the above method. Then the L.t.s. $\mathbb{B}_{-1} \oplus \mathfrak{S}_{1}$ in $\mathbb{G}$ with the $\varepsilon$-structure $\tau$ is isomorphic to $\mathfrak{I}\left(\mathbb{S}_{-1}\right)$ by the mapping $a+\tau_{-1}(x) \rightarrow a+x$. Therefore, if we assume the semi-simplicity of $\mathbb{B}$, the two Lie algebras $\mathbb{B}$ and $\mathfrak{L}\left(\mathbb{S}_{-1}\right)$ are isomorphic.

EXAMPLES. For the graded simple Lie algebra of second order with a 1-structure in $\mathbb{S}_{-1} \oplus \mathbb{S}_{1}$, we know the models constructed by Tits-Koecher [5] and I. L. Kantor [4]. However, the Tits-Koecher's models are of $\mathfrak{B}_{ \pm 2}=\{0\}$. For -1 -structure, there are the models by H. Freudenthal [2] and B. N. Allison [1].

Remark. Any automorphism $\tau$ of the L.t.s. $\mathbb{S}_{-1} \oplus \mathfrak{S}_{1}$ can be canonically extended to an automorphism $\tau$ of the Lie algebra (\$s. In fact, under the notation of (1.3), we can define the automorphism $\tau: \mathbb{S} \rightarrow \mathbb{S}$ by $\tau(D+E)=\tau D \tau^{-1}$ $+\tau(E)$ for $D+E \in\left(\mathbb{S}_{-2} \oplus \mathbb{S}_{0} \oplus \mathbb{S}_{2}\right) \oplus\left(\mathbb{S}_{-1} \oplus \mathbb{S}_{1}\right)$. Hence, any 1-structure $\tau$ can be extended to an involutive automorphism of $\mathbb{B}$ and -1 -structure $\tau$ becomes an automorphism with $\tau^{4}=1$ where $\tau^{2}=1$ in $\mathbb{S}_{-2} \oplus \mathbb{S}_{0} \oplus \mathbb{S}_{2}$.

\section{$\S 2$. Simplicity of $\mathfrak{L}(V)$.}

Let $V$ be a Kantor (resp. Freudenthal) system with a triple product $L(a, b) c$ such that $L(V, V) V \neq\{0\}$ and let $\mathscr{L}(V)$ be the standard imbedding (Lie algebra) of the L.t.s. $\mathfrak{I}(V)$. Usually, $V$ is said to be simple if $V$ has no subspaces $\{W\}$ except $\{0\}$ and $V$ such that $L(W, V) V \subset W, L(V, W) V \subset W$ and $L(V, V) W \subset W$.

Proposition 2. If $\mathfrak{L}(V)$ is simple, $V$ is simple. Conversely, if $V$ is simple, $\mathfrak{L}(V)$ is semi-simple. 
Proof. Put $V=\mathfrak{B}_{-1}$. We assume that $\mathfrak{A}$ is any ideal of $\mathfrak{S}_{-1}$. Then $\mathfrak{A} \oplus \tau(\mathfrak{A})$ is an ideal of the L.t.s. $\mathfrak{I}\left(\mathbb{S}_{-1}\right)$ where $\tau_{-1}=1$ and $\tau_{1}=\varepsilon$ with $\varepsilon=1$ (resp. -1 ). Since $\mathfrak{I}\left(\mathbb{S}_{-1}\right)$ is simple by the simplicity of $\mathfrak{L}\left(\mathbb{S}_{-1}\right)$, we have $\mathfrak{A}=\{0\}$ or $\mathbb{S}_{-1}$. This means that $\mathbb{S}_{-1}$ is simple.

Conversely, we assume that $\mathscr{S}_{-1}$ is simple. For the L.t.s. $\mathfrak{I}=\mathfrak{I}\left(\mathbb{S}_{-1}\right)$ of $\mathfrak{L}\left(\mathfrak{S}_{-1}\right)$, it always holds $\mathfrak{R}\left(\mathfrak{L}\left(\mathbb{S}_{-1}\right)\right)=\mathfrak{R}(\mathfrak{I}) \oplus[\Re(\mathfrak{I}), \mathfrak{I}]$ where $\mathfrak{R}\left(\mathfrak{L}\left(\mathbb{S}_{-1}\right)\right)$ and $\mathfrak{R}(\mathfrak{I})$ are the radicals of $\mathfrak{L}\left(\mathbb{S}_{-1}\right)$ and $\mathfrak{I}\left(\mathbb{S}_{-1}\right)$ respectively (cf. [6]). Under the notation of (1.3), since the mapping $\theta=\left(\begin{array}{cc}1 & 0 \\ 0 & -1\end{array}\right)$ of $\mathfrak{I}$ makes the radical $\Re(\mathfrak{I})$ invariant and $\tau$ is an automorphism of $\mathfrak{I}$, there is an ideal $\mathfrak{A}$ of $\mathfrak{B}_{-1}$ such that $\mathfrak{R}(\mathfrak{I})=$ $\mathfrak{X} \oplus \tau(\mathfrak{U}) \subset \mathfrak{I}$. Because $\mathfrak{S}_{-1}$ is simple, if we assume $\mathfrak{A}=\mathbb{S}_{-1}$, we have $\mathfrak{L}\left(\mathbb{S}_{-1}, \mathbb{S}_{-1}\right) \mathbb{S}_{-1}=\mathbb{S}_{-1}$ and $\mathfrak{I} \subset \mathfrak{R}(\mathfrak{I})^{(n)}\left(=\left[\mathfrak{I}, \mathfrak{R}(\mathfrak{I})^{(n-1)}, \mathfrak{R}(\mathfrak{I})^{(n-1)}\right]\right.$ where $[,$,$] is$ the triple product of the L.t.s. $\mathfrak{I})$ for any natural number $n$. But this contradicts $\mathfrak{S}_{-1} \neq\{0\}$. Therefore $\mathfrak{A}=\{0\}$, i. e., $\mathfrak{R}(\mathfrak{I})=\mathfrak{R}\left(\mathfrak{L}\left(\mathbb{S}_{-1}\right)\right)=\{0\}$.

\section{$\S 3$. Isomorphisms of $\mathfrak{L}(V)$.}

Two triple systems $V_{1}, V_{2}$, having triple products $L_{1}(a, b) c, L_{2}(x, y) z$ respectively, are weakly isomorphic if there are two one-to-one onto mappings $P, Q: V_{1} \rightarrow V_{2}$ such that $P L_{1}(a, b) c=L_{2}(P a, Q b) P c$ and $Q L_{1}(a, b) c=L_{2}(Q a, P b) Q c$ for $a, b, c \in V_{1}$ where we use the notation $P a$ instead of $P(a)$. Then we have $P K_{1}(a, b) c=K_{2}(P a, P b) Q c$ and $Q K_{1}(a, b) c=K_{2}(Q a, Q b) P c$ where $K_{i}(a, b) c=$ $L_{\imath}(a, c) b-L_{i}(b, c) a(i=1,2)$.

Proposition 3. Two standard imbedding (Lie algebra) $\mathfrak{L}\left(\mathbb{S}_{-1}\right)$ and $\mathfrak{L}\left(\mathbb{S}_{-1}^{\prime}\right)$ are isomorphic (by an isomorphism preserving the grading) if and only if two triple systems $\mathbb{S}_{-1}$ and $\mathbb{S}_{-1}^{\prime}$ are weakly isomorphic. If $\sigma$ is a grade-preserving isomorphism of $\mathfrak{L}\left(\mathbb{S}_{-1}\right)$ to $\mathfrak{L}\left(\mathbb{S}_{-1}^{\prime}\right)$, we can have $P=\sigma \mid \mathbb{S}_{-1}$ (the restriction of $\sigma$ to $\left.\mathbb{S}_{-1}\right)$ and $Q=\tau^{\prime-1} \sigma \tau \mid \mathbb{S}_{-1}$ where $\tau$ and $\tau^{\prime}$ are $\varepsilon$-structures in $\mathfrak{I}\left(\mathbb{S}_{-1}\right)$ and $\mathfrak{I}\left(\mathbb{S}_{-1}^{\prime}\right)$ respectively.

Proof. If $\mathbb{S}_{-1}$ and $\mathbb{B}_{-1}^{\prime}$ are weakly isomorphic, there is an isomorphism $\sigma: \mathfrak{I}\left(\mathbb{S}_{-1}\right) \rightarrow \mathfrak{I}\left(\mathbb{S}_{-1}^{\prime}\right)$ with respect to the triple product of the L.t.s. which is defined by $\sigma(a+\tau(b))=P a+\tau^{\prime}(Q b)$ for $a, b \in \mathbb{S}_{-1}$. And, this $\sigma$ can be canonically extended to an isomorphism of the standard imbedding (Lie algebra) by the same method as the Remark of Theorem 1.

Conversely, let $\sigma$ be a grade-preserving isomorphism of $\mathfrak{L}\left(\mathbb{S}_{-1}\right)$ to $\mathfrak{L}\left(\mathbb{S}_{-1}^{\prime}\right)$. When we put $P=\sigma \mid \mathbb{S}_{-1}$ and $Q=\tau^{\prime-1} \sigma \tau \mid \mathbb{S}_{-1}$, we have $P L_{1}(a, b) c=\sigma[[a, \tau(b)], c]$ $=[[\sigma(a), \sigma \tau(b)], \sigma(c)]=\left[\left[\sigma(a), \tau^{\prime} \tau^{\prime-1} \sigma \tau(b)\right], \sigma(c)\right]=L_{2}(P a, Q b) P c$ for $a, b, c \in \mathbb{S}_{-1}$. The other relation $Q L_{1}(a, b) c=L_{2}(Q a, P b) Q c$ can be proved similarly. 


\section{$\S 4$. A duality.}

There is a simple connection between the Kantor systems and the Freudenthal systems.

THEOREM 4. Let $V$ be a Kantor (resp. Freudenthal) system with a triple product $L(a, b) c$. If there exist an automorphism $\Phi$ of $V$, i.e., $\Phi(L(a, b) c)=$ $L(\Phi(a), \Phi(b)) \Phi(c)$ for $a, b, c \in V$, such that $\Phi^{2}=-1, V$ becomes a Freudenthal (resp. Kantor) system with respect to the new triple product $L(a, \Phi(b)) c$ (resp. $-L(a, \Phi(b)) c)$. This mapping $\Phi$ is also an automorphism for the new product.

EXAMPLE. Let $V(=(5)$ be the Cayley algebra over the complex numbers C. Then $V$ is a Kantor system by the triple product $L(a, b) c=a(\bar{b} c)+c(\vec{b} a)-b(\bar{a} c)$ for $a, b, c \in V$, where - is the usual conjugation of $V$, and $\mathscr{L}(V)$ is a simple Lie algebra of type $\boldsymbol{F}_{4}$. In this case, the right multiplication $\Phi$ is an automorphism for the triple product $a(\bar{b} c)$ in $V$ where $\Phi(x)=x v$ for any $x \in V$ and some fixed $v \in V$ with $\operatorname{tr}(v)=0$ and $v v=-1$. Therefore $\Phi$ is also an automorphism with respect to the product $L(a, b) c$ and $V$ becomes a Freudenthal system by the product $L(a, b v) c=a((\overline{b v}) c)+c(\overline{(b v}) a)-\langle b v)(\bar{a} c)$ for $a, b, c \in V$ (cf. $[3])$.

\section{REFERENCES}

[1] B. N. Allison, A construction of Lie algebras from $J$-ternary algebras, Amer. J. Math., 98 (1976), 285-294.

[2] H. Freudenthal, Beziehungen der $E_{7}$ und $E_{8}$ zur Oktavenebene. II, Indag. Math., 16 (1954), 363-368.

[3] W. Hein, A connection between Lie algebras of type $F_{4}$ and Cayley algebras, Indag. Math., 38 (1976), 419-425.

[4] I. L. Kantor, Models of exceptional Lie algebras, Soviet Math. Dokl., 14 (1973), $254-258$.

[5] M. Koecher, Imbedding of Jordan algebras into Lie algebras. I, Amer. J. Math., 89 (1967), 787-816.

[6] W. G. Lister, A structure theory of Lie triple systems, Trans. Amer. Math. Soc., 72 (1952), 217-242.

[7] O. Loos, Symmetric spaces I, Benjamın, New York, 1969.

[8] K. YAMAGUTI, Remarks on characterizations of the points and symplecta in the metasympletic geometry, Mem. Gen. Ed., Kumamoto Univ., Ser. Nat. Sci., 11 (1976), 1-8.

Kumamoto Institute of Techinology

IKeda, Kumamoto 860

JAPAN 